SCMT4

Las Vegas, USA, August 7-11, 2016

\title{
Molecular Dynamics (MD) Model Generation for the Graphite Nanoplatelets Modified Asphalt
}

\author{
Hui Yao ${ }^{1 a}$, Qingli Dai ${ }^{1 b}$, and Zhanping You ${ }^{1 \mathrm{c}}$ \\ ${ }^{1}$ Department of Civil and Environmental Engineering, Michigan Technological University, Houghton, \\ Michigan,49931,USA. ${ }^{1 a}$ E-mail: <huiyao@mtu.edu>, ${ }^{1 b}$ E-mail: 〈qingdai@mtu.edu>, ${ }^{1 c}$ E-mail: \\ <zyou@mtu.edu>
}

\begin{abstract}
Molecular dynamics is a new computational method for material analysis by studying the physical movements of atoms and molecules. It is expected to have a great impact on material engineering. The discovery of advanced materials would be expedited and the indirect cost of the materials will be saved in different areas of industry. The purpose of this study is to generate the modified asphalt model with multilayer graphite xGNP nanoplatelets using the Molecular Dynamics (MD) method. The common graphite model represents the xGNP particles. The base asphalt is composed of three components: asphaltenes, aromatics, and saturates. The xGNP model was added to the base asphalt model by molar mass, and the xGNP modified asphalt model was generated after the energy minimizations and optimizations for a few thousand steps. The Isothermal-isobaric ensemble (NPT ensemble) was employed to compress the xGNP modified asphalt model. The energy, temperature, pressure, and density were predicted and calculated for the xGNP modified asphalt model compared to the base asphalt model. The densities of the base and modified asphalt models are approximated to those of experimental and reference data.
\end{abstract}

\section{INTRODUCTION}

Molecular Dynamics (MD) is a new kind of analysis tool for material simulations based on Newton's law. It is commonly used in physical and chemical engineering, but a few studies use it in civil engineering materials. The MD method was used to analyze the materials on an atomic scale, and it can provide a new viewpoint for researchers. It is also a challenge to conduct this kind of simulation to understand fundamental mechanisms of the material behaviors. It is important to properly choose the simulation size and reasonable time for material simulations. In addition, when the economy develops, the cost of materials and labor increases. During the development of new material, the extensive preliminary data is required to determine if the new material works or not. That is a challenge for materials in different research areas, and it aggravates the difficulty and cost during development. A new computational analysis tool, Molecular Dynamics (MD), has a significant impact on the trajectory of the material area. The use of the MD method improves the material discovery process, expedites the selections, and saves the cost of materials and labor. More importantly, the success rate will be improved during material development.

Usually the Large-scale Atomic/Molecular Massively Parallel Simulator (LAMMPS) (Plimpton, 1995) and the Monte Carlo for Complex Chemical Systems (MCCCS) program (Martin, 2013) are used for MD simulations. The base asphalt model was simulated by three components, and the properties of the base asphalt model were computed and compared to laboratory data including the density, glass transition 
temperature, viscosity, bulk modulus, thermal expansion coefficient, and isothermal compressibility, etc. The properties of the base asphalt calculated by simulations are still different from the experimental data (Yao et al., 2016, Zhang and Greenfield, 2007). The relationship between the asphalt and aggregate was also analyzed through the MD method, and the adhesion energy of this interface was calculated (Yao et al., 2015).

Few researchers utilized the MD method to study the modified asphalt model, and also analyze the properties of the modified asphalt model. In this simulation, the common graphite model represents the multi-layer graphite xGNP nanoplatelets for the modifiers in the modification. The base asphalt model was based on the previous study (Yao et al., 2016), and the Amber Cornell Extension Force Field (ACEFF) and Electrostatic Potential (ESP) charges were adopted for the components and asphalt models. The system parameters were monitored during the Isothermal-isobaric ensemble (NPT ensemble), such as energy, pressure, temperature and density of the base and xGNP modified asphalt models.

\section{FORCE FIELD}

A force field, is a mathematical function to define the energy in the MD systems. Different force fields may be suitable for different materials. In this simulation, the Amber Cornell Extension Force Field was adopted in the asphalt models (equation (1)) and the Isothermal-isobaric ensemble (NPT ensemble) was used to compress the asphalt models.

$E_{\text {total }}=\sum_{\text {bonds }} K_{r}\left(r-r_{e q}\right)^{2}+\sum_{\text {angles }} K_{\theta}\left(\theta-\theta_{e q}\right)^{2}+\sum_{\text {dihedrals }} \frac{V_{n}}{2}[1+\cos (n \varphi-\gamma)]+\sum_{i<j}\left[\frac{A_{i j}}{R_{i j}^{12}}-\right.$ $\left.\frac{B_{i j}}{R_{i j}^{6}}+\frac{q_{i} q_{j}}{\epsilon R_{i j}}\right]$

In Equation 1 above, $r_{e q}$ and $\theta_{e q}$ are structural parameters; $K_{r}$ and $K_{\theta}$ are the force coefficients; $\mathrm{n}$ and $\gamma$ are the parameters for dihedrals; A, B and q are the non-bonded potentials.

\section{OBJECTIVES AND SCOPES}

This study aims to simulate the xGNP modified asphalt model using the MD method and calculate the densities of the xGNP modified asphalt model. The components of the base asphalt were based on the author's previous study (Yao et al., 2016), and a common graphite model was adopted for the xGNP model. The Amber Cornell Extension Force Field and Electrostatic Potential (ESP) charges were used for these components. The xGNP modified asphalt model was produced, and the densities of these models were computed.

\section{MODEL GENERATION}

Based on recent work of the authors (Yao et al., 2016), three components for the base asphalt include asphaltenes, aromatics, and saturates, and a ratio of 5:27:41 was adopted. In this base asphalt model, the 1, 7-Dimethylnaphthalene and docosane were selected for aromatics and saturates, respectively (asphaltene: $\mathrm{C}_{64} \mathrm{H}_{52} \mathrm{~S}_{2}$, aromatic: $\mathrm{C}_{12} \mathrm{H}_{12}$, and saturate: $\mathrm{C}_{22} \mathrm{H}_{46}$ ). In the experimental tests, $x G N P$ graphene nanoplatelets manufactured by XG Sciences Inc. was used for the modification of the asphalt and the micro-image of the xGNP particles (Figure 1a) was observed by the field emission scanning electron microscope (FE-SEM). The content of the xGNP nanoplatelets in the modification is around $2 \%$ by weight of the base asphalt. The xGNP particles were added to the base asphalt at $145^{\circ} \mathrm{C}$, and the mixture was sheared in the high shear machine for around two hours. Similarly, in this simulation, the xGNP model (Figure 1b) represents the xGNP particles, and $2 \%$ xGNP particles were added to the base asphalt model by molar mass. The xGNP modified asphalt model $\left(\mathrm{C}_{6184} \mathrm{H}_{9880} \mathrm{~S}_{40}\right)$ was created (Figure 2c). 


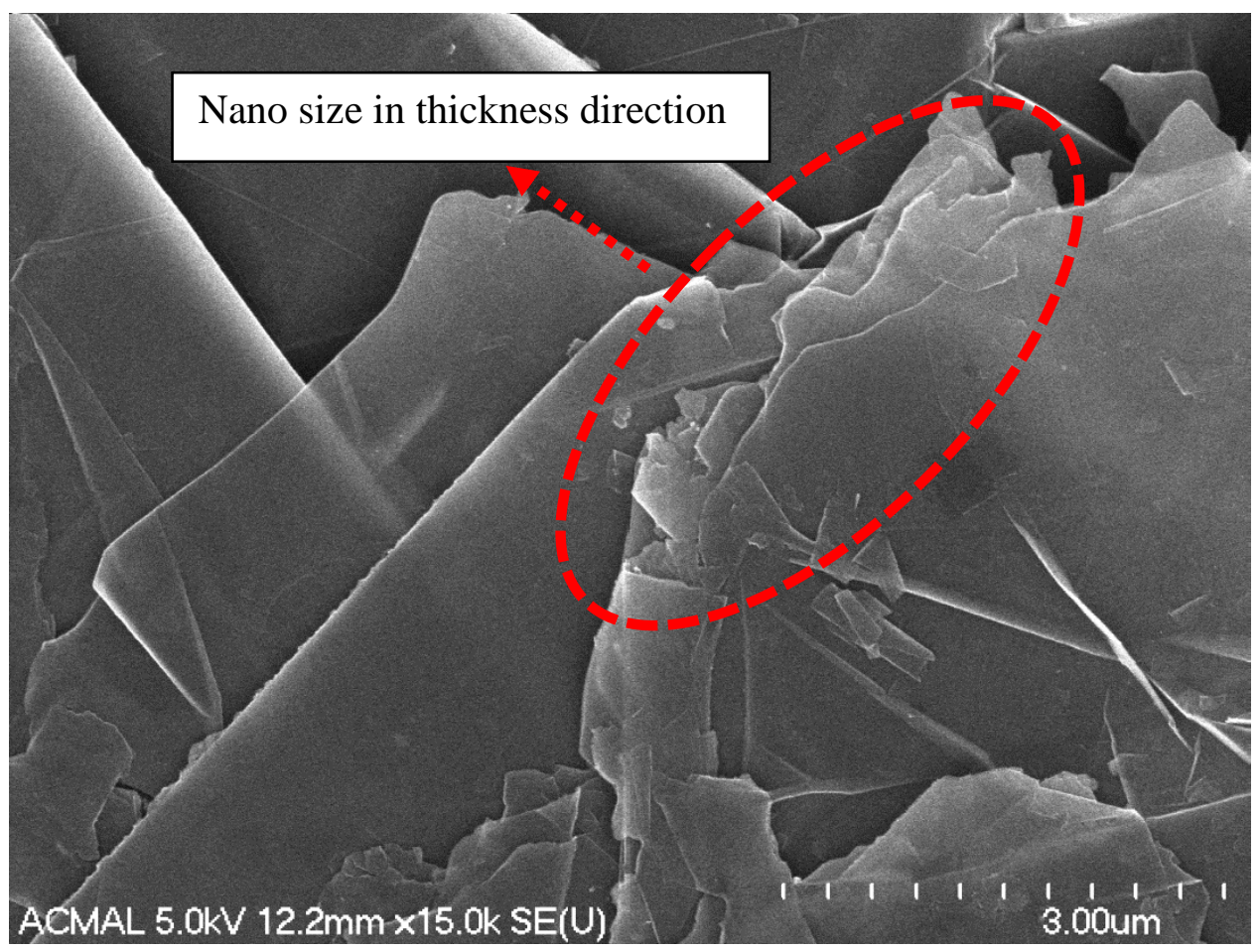

(a) micro-image of xGNP nanoplatelets

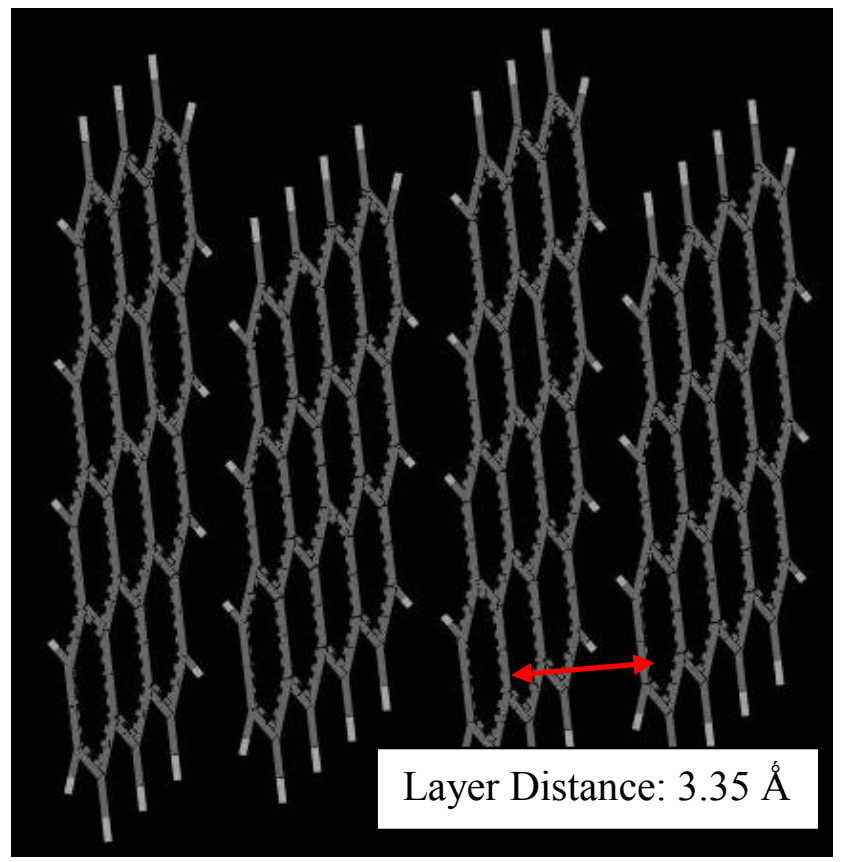

(b)xGNP model (white wire: hydrogen atom; grey wire: carbon atom)

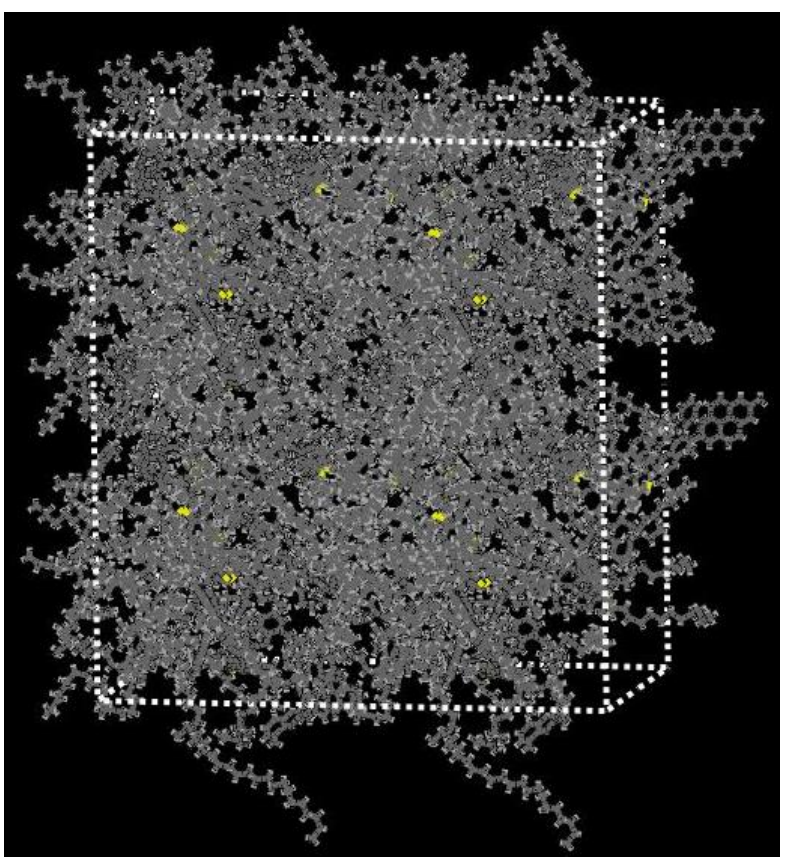

(c) MD structure of $x$ GNP modified asphalt model (white wire: hydrogen atom; grey wire: carbon atom and yellow wire: sulfur atom)

Figure 1. Micro-image of multi-layer xGNP particles, MD structures of xGNP and the modified asphalt models 


\section{DENSITY OF XGNP MODIFIED ASPHALT MODEL}

The $\mathrm{xGNP}$ modified asphalt model was prepared, and the energy minimizations and optimizations were conducted to reach a stable state. This model was compressed and relaxed using NPT ensemble at the conditions of $25^{\circ} \mathrm{C}$ and 1 atm pressure. The simulation results are shown in Figure 2, including system energy during NPT ensemble, temperatures, pressures and densities in the base and xGNP modified asphalt systems.

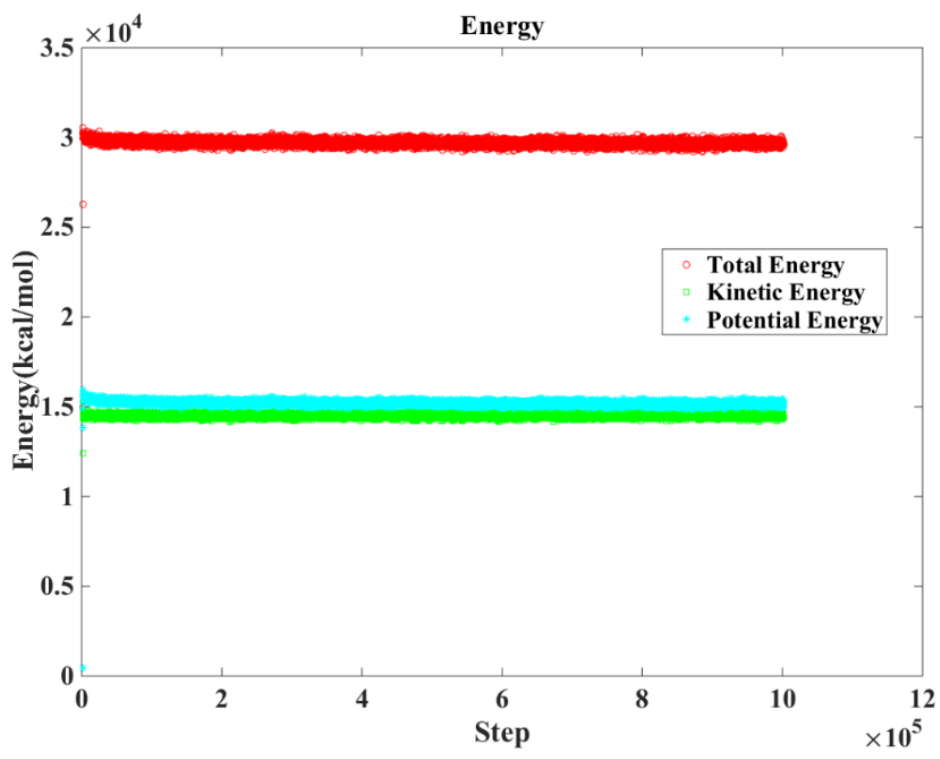

(a) Different energies of xGNP modified asphalt model

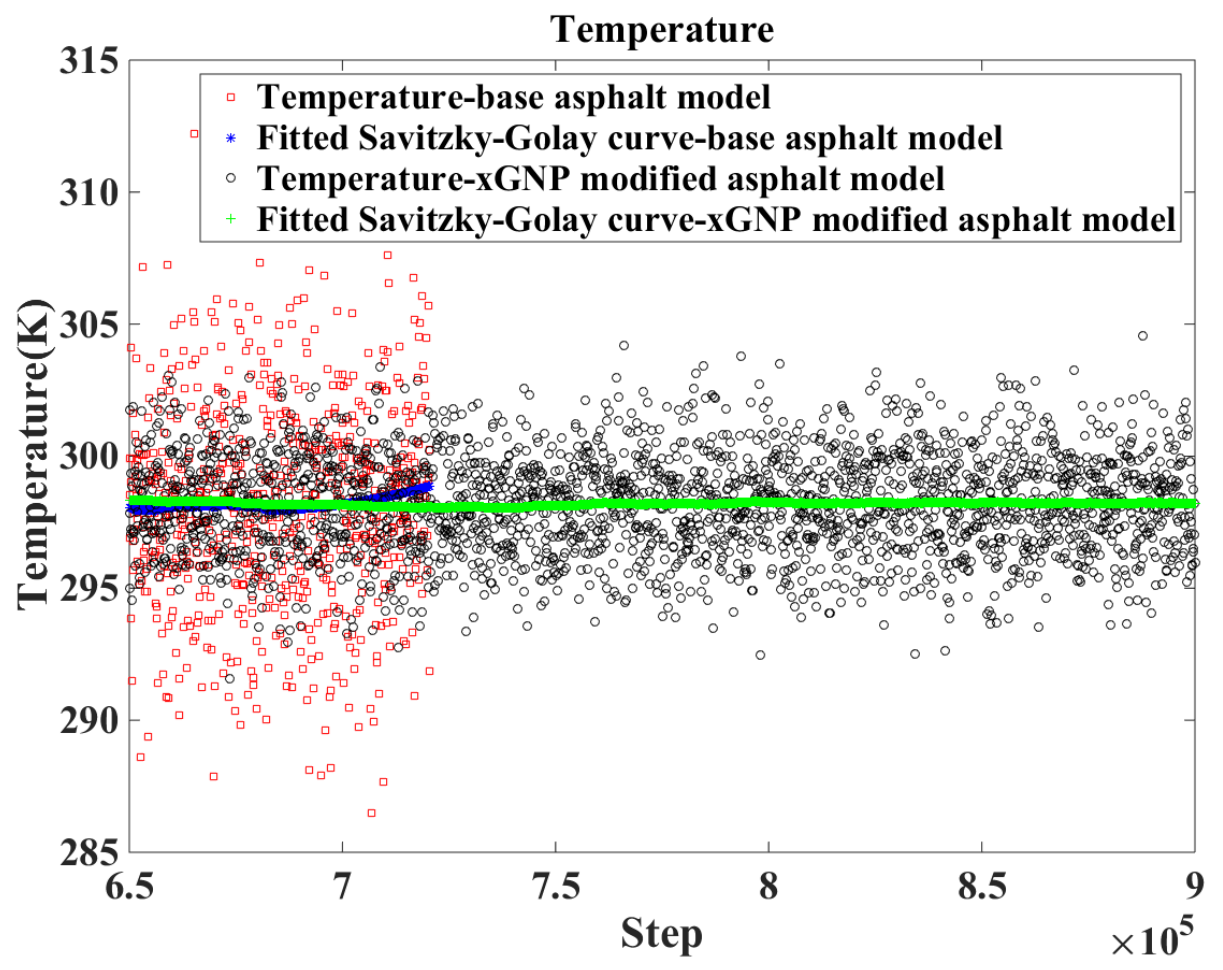

(b) Temperatures of the xGNP modified asphalt system through these steps 


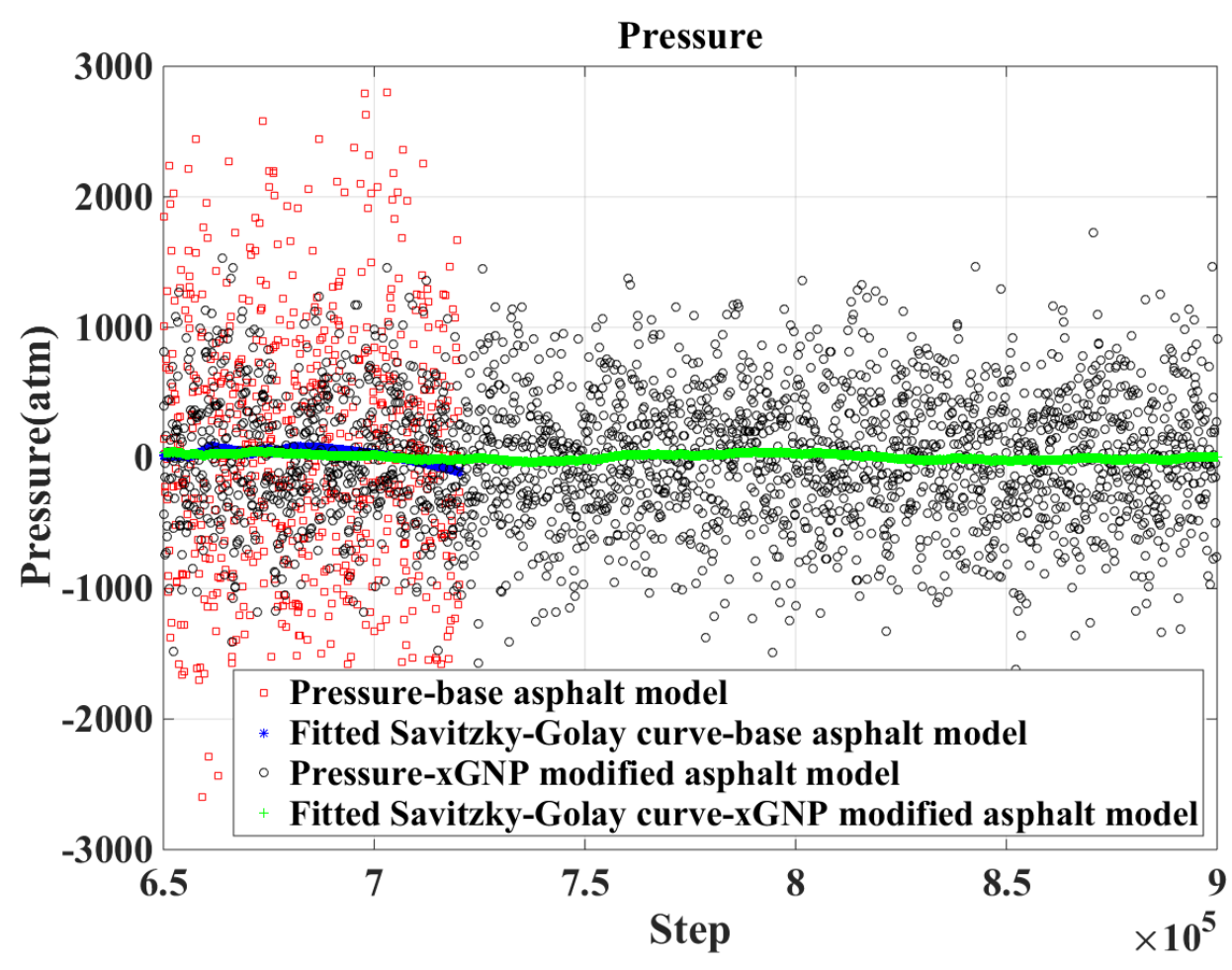

(c) Pressures of the xGNP modified asphalt model

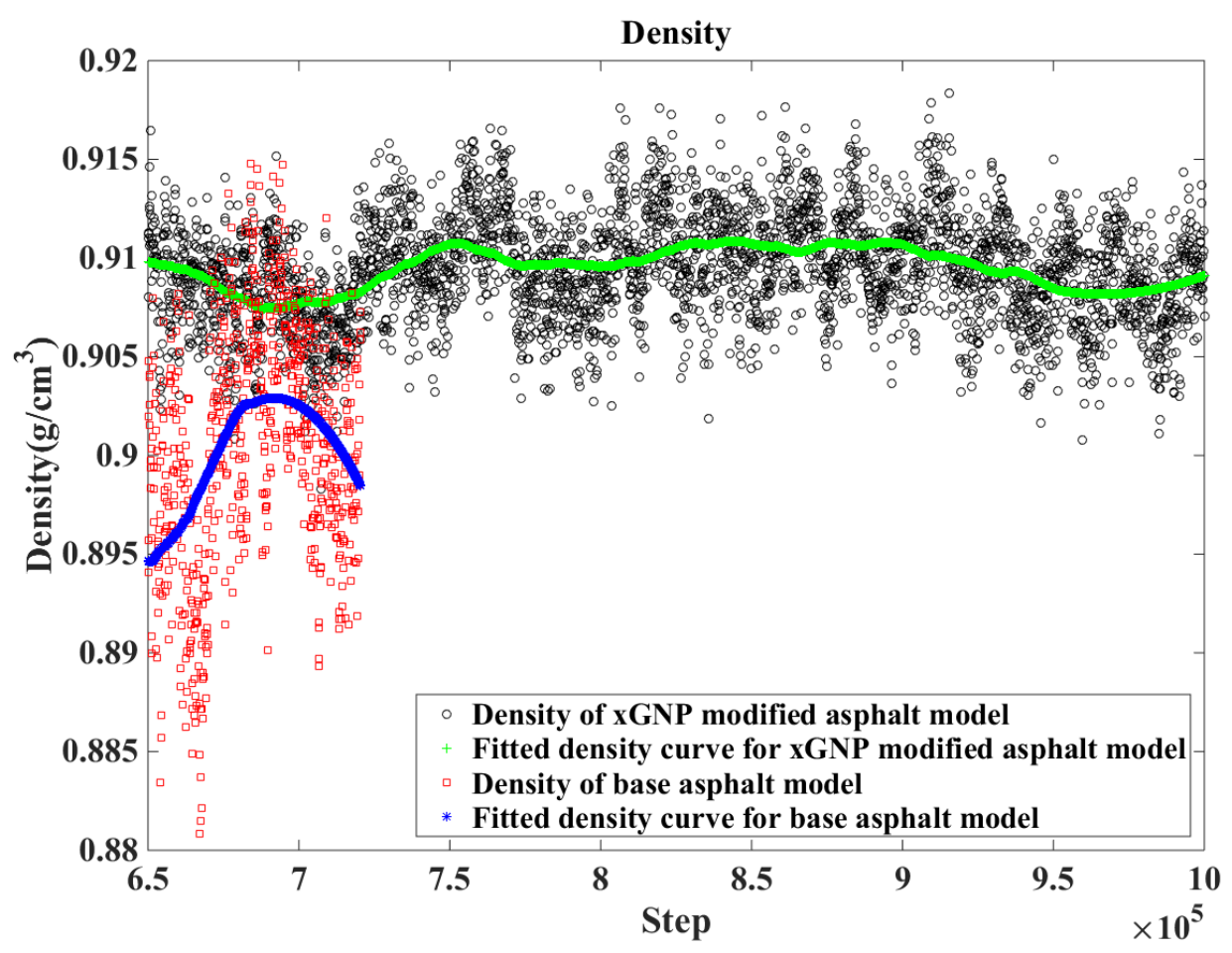

(d) Densities of the xGNP modified asphalt model

Figure 1. Density curves of the control and xGNP modified asphalt models 
Figure 2a displays the energies in the asphalt systems during the NPT ensemble, and it indicates that the total energy of the xGNP modified asphalt model reaches equilibrium after energy minimizations and NPT ensemble. Figure $2 b$ and $2 c$ show the system temperatures and pressures of the base and xGNP modified asphalt models during the NPT simulations. These systems have a relatively stable state after a few simulations. The Savitzky-Golay filter was employed to demonstrate the moving average. The system temperature was kept around $298 \mathrm{~K}$ (room temperature), and the system pressure was maintained at around $1 \mathrm{~atm}$. It is consistent with the input requirements. Figure $2 \mathrm{~d}$ shows the density trends of the base and xGNP modified asphalt models, and these trends were also fitted by the Savitzky-Golay filter (Savitzky and Golay, 1964) with a span of $10 \%$. The densities of the xGNP asphalt model are higher than the base asphalt's. It can be inferred that the xGNP model in the base asphalt model increases the density since more molecular mass was added. The density of the asphalt was tested in the laboratory, and it is around $0.95-1.05 \mathrm{~g} / \mathrm{cm}^{3}$ (Greenfield and Zhang, 2009, Li and Greenfield, 2014, Zhang and Greenfield, 2007). The densities of the asphalt models in this study are close to those of the experiments and references (Zhang and Greenfield, 2007).

\section{CONCLUSION}

The Molecular Dynamics (MD) method emerged as a new analysis tool for the materials, and it is beneficial to analyze the materials on the atomic scale to fundamentally understand the materials from a nano scale. In this simulation, the base asphalt model was generated based on the previous study, and the MD model for multi-layer graphite xGNP nanoplatelets was also created. The xGNP modified asphalt model was prepared at a specific condition, and the energy minimization and optimization were conducted for the stable structure. The NPT ensemble was used to compress the model and calculate the density of the xGNP modified asphalt model. From the simulation results, the condition of the MD simulation was the same as the requirements, and the densities of the base and modified asphalt models are approximately those of the experiments or references. In addition, an xGNP modified asphalt model was generated and verified by density, one of the physical properties. This preliminary process of MD study brought a potential to validate whether the modifier (xGNP nanoplatelets) can improve the performance of asphalt or not. It is also beneficial that the modification mechanism was disclosed during MD simulations. It could save tremendous laboratory costs and labor, as well as the waste of materials. It is important for industry to screen materials or modifiers during the construction or materials research and development.

\section{ACKNOWLEDGEMENT}

The authors appreciate the financial support of the U.S. National Science Foundation (NSF) under grant 1300286. The computational studies were performed using a computer cluster (Superior research center) at Michigan Technological University. Any opinion, finding, and conclusion expressed in this paper are those of the authors and do not necessarily represent the view of any organization.

\section{REFERENCES}

Greenfield, M. L. \& Zhang, L. 2009. Final Report-Developing Model Asphalt Systems Using Molecular Simulation. Uritc Project No. 000216. Department Of Chemical Engineering, University Of Rhode Island, Kingston, Ri 02881: University Of Rhode Island Transportation Center.

Li, D. D. \& Greenfield, M. L. 2014. Chemical Compositions Of Improved Model Asphalt Systems For Molecular Simulations. Fuel, 115, 347-356.

Martin, M. G. 2013. Mcccs Towhee: A Tool For Monte Carlo Molecular Simulation. Molecular Simulation, $39,1212-1222$.

Plimpton, S. 1995. Fast Parallel Algorithms For Short-Range Molecular Dynamics. Journal Of Computational Physics, 117, 1-19. 
Savitzky, A. \& Golay, M. J. E. 1964. Smoothing And Differentiation Of Data By Simplified Least Squares Procedures. Analytical Chemistry, 36, 1627-1639.

Yao, H., Dai, Q. \& You, Z. 2015. Chemo-Physical Analysis And Molecular Dynamics (Md) Simulation Of Moisture Susceptibility Of Nano Hydrated Lime Modified Asphalt Mixtures. Construction And Building Materials, 101, Part 1, 536-547.

Yao, H., Dai, Q. \& You, Z. 2016. Molecular Dynamics Simulation Of Physicochemical Properties Of The Asphalt Model. Fuel, 164, 83-93.

Zhang, L. \& Greenfield, M. L. 2007. Analyzing Properties Of Model Asphalts Using Molecular Simulation. Energy \& Fuels, 21, 1712-1716. 